\title{
A Recurrently Generated Overlay Architecture for Rapid FPGA Application Development
}

\author{
David Wilson, Greg Stitt \\ University of Florida \\ Gainesville, FL, USA \\ d.wilson@ufl.edu,gstitt@ece.ufl.edu
}

\author{
James Coole \\ Cisco Systems \\ Research Triangle Park, NC, USA \\ jcoole@cisco.com
}

\begin{abstract}
Recent FPGA research has increasingly focused on overlays - virtual coarse-grained architectures - to address widely known application-design productivity problems such as lengthy compilation times and a lack of portability. However, existing overlay research has not yet been adopted due to several key limitations: 1) a general focus on datapath-centric applications with little control logic, and/or 2) manual creation of overlays for a given application or domain. Ideally, FPGA design tools would automatically generate specialized overlays for any application. In this paper, we present a first step towards this ideal goal by integrating datapath-centric overlays with finite-statemachine overlays, which we recurrently generate as application characteristics and resource requirements change during application development. By generating overlays capable of supporting arbitrary controllers and datapaths, our approach enables rapid development of numerous FPGA applications, while potentially providing a target architecture to enable high-level synthesis that is comparable in productivity to compilers for microprocessors and GPUs. We demonstrate that our proposed overlay architecture can enable compilation times that are over $100,000 \times$ faster than register-transfer-level designs, with overheads comparable to existing overlays. For iterative development, we show recurrent overlay generation provides faster development cycles than traditional FPGA development after only two design iterations, which improves further for additional iterations by amortizing overlay generation across multiple rapid virtual compilations and reconfigurations.
\end{abstract}

\section{INTRODUCTION}

Despite attractive performance and energy in important application domains, field-programmable gate arrays (FPGAs) commonly go unused due to application-design productivity that is significantly worse than other devices [6].

\footnotetext{
Permission to make digital or hard copies of all or part of this work for personal or classroom use is granted without fee provided that copies are not made or distributed for profit or commercial advantage and that copies bear this notice and the full citation on the first page. Copyrights for components of this work owned by others than ACM must be honored. Abstracting with credit is permitted. To copy otherwise, or republish, to post on servers or to redistribute to lists, requires prior specific permission and/or a fee. Request permissions from Permissions@acm.org.

HEART 2018, June 20-22, 2018, Toronto, ON, Canada

(C) 2018 Association for Computing Machinery. ACM ISBN 978-1-4503-6542-0/18/06 ..\$15.00 https://doi.org/10.1145/3241793.3241797
}

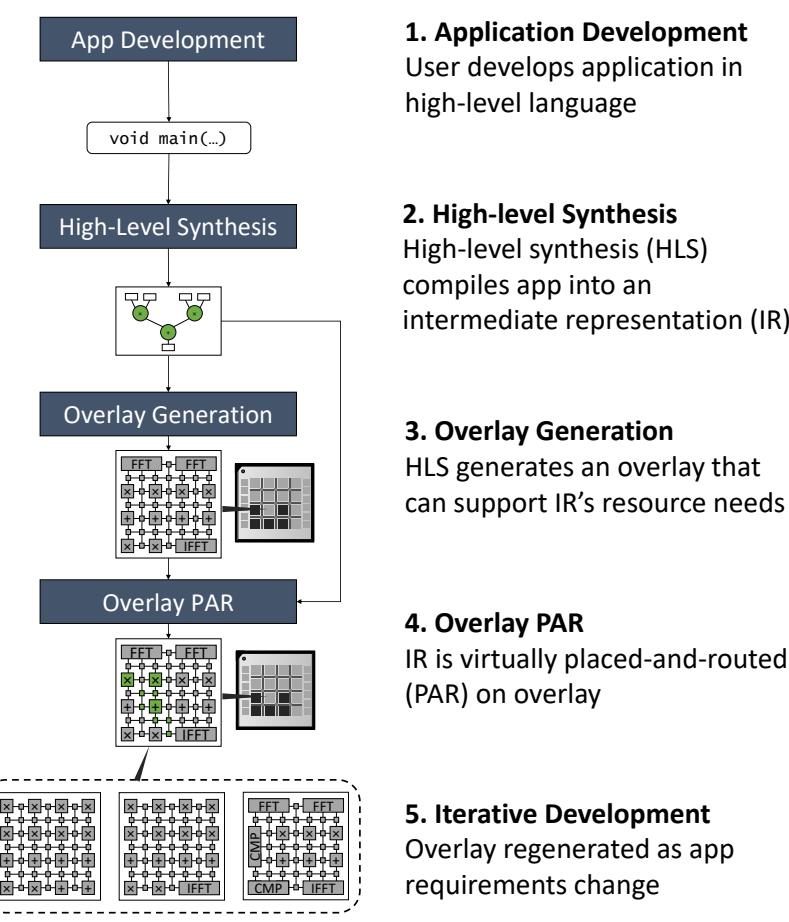

Figure 1. Overview of overlay-based design flow.

Although high-level synthesis (HLS) hides many registertransfer-level (RTL) challenges, FPGA application design still suffers from prohibitive productivity problems, such as lengthy compilation that can take on the order of hours or even days [8]. Other productivity problems include limited portability and code reuse, and low-level debugging.

Recent FPGA research has aimed to minimize lengthy compilation with coarse-grained virtual architectures implemented atop FPGAs, commonly referred to as overlays. By abstracting the fine-grained FPGA as a fabric of coarsegrained resources, the overlay presents a reconfigurable architecture that can rapidly alter its functionality with a small set of configuration data. Recent overlay studies have shown place-and-route speedups of over 1,000 $\times$ compared to vendor tools [2], with some as high as 10,000× [3].

Despite these compilation speedups, overlays are not ready for widespread adoption due to several key limitations. First, most overlay studies support a small subset of possible application functionality, typically focusing on datapathcentric functions with minimal control requirements (e.g., [8]). Although such overlays are appropriate for the targeted applications, without virtual control components, overlays are not applicable to more general FPGA development. Recent work has addressed control limitations by introducing 


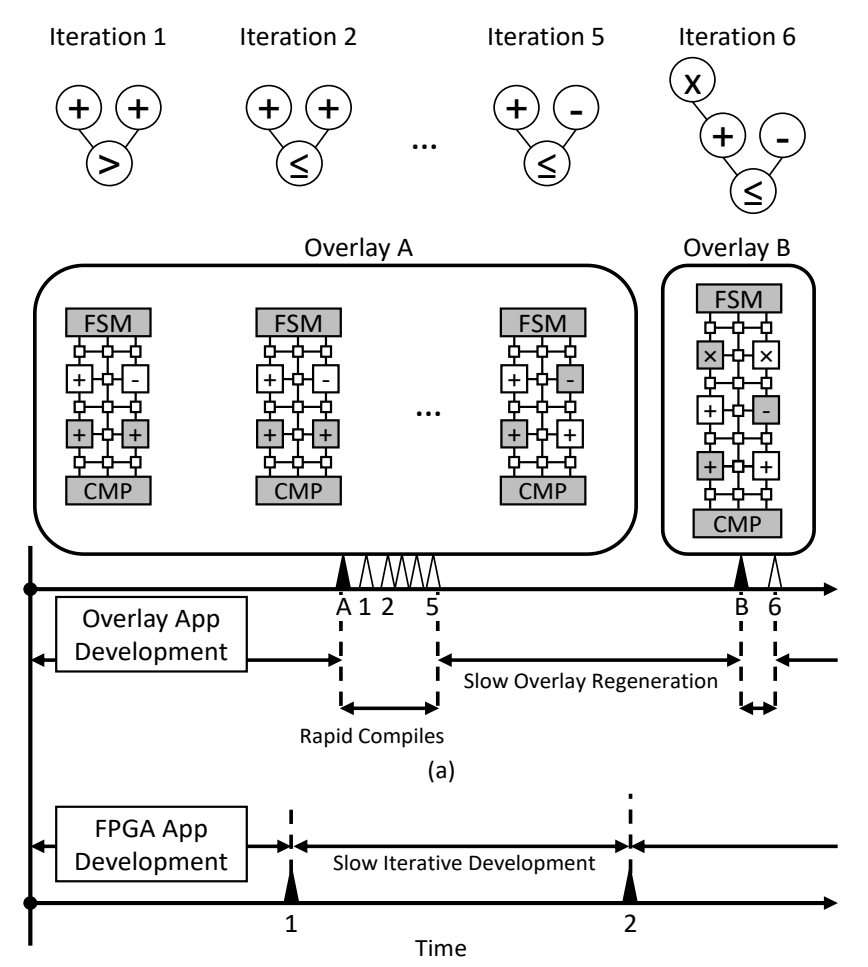

(b)

Figure 2. An illustration of rapid application development using (a) occasional recurrent generation of overlays when application requirements shift significantly, compared to (b) traditional FPGA application development with long compilation times.

overlay architectures that support virtual finite state machines (FSMs) [1], [9]. While those approaches are effective solutions to FSMs by themselves, the approaches do not address integration between control and datapath overlays that is needed for more general development.

In addition to technical limitations, many existing overlay approaches are limited practically by the use of manually created overlay architectures for a specific application or domain. Due to the wide range of FPGA applications, and the numerous Pareto-optimal tradeoffs for implementing a single application, manually creating efficient overlays for general-purpose FPGA development is infeasible. Although some approaches automatically specialize an overlay based on application code [3], those approaches suffer from the previous limitation of supporting limited functionality.

Ideally, FPGA design tools would transparently integrate overlays into general FPGA application development by automatically generating a flexible overlay. Figure 1 illustrates an example use case that provides a target architecture for HLS to enable rapid compilation comparable to microprocessors and graphics-processing units. Instead of mapping application functionality onto an FPGA or a manually designed overlay, HLS would generate a custom overlay (or select an existing one) to implement the circuit. Although overlay generation suffers from long compiles similar to existing FPGA synthesis, our approach amortizes those lengthy compiles over numerous design iterations where the overlay provides $100,000 \times$ faster compilation.
In this paper, we present a first step towards this ideal goal by automatically generating an integrated control and datapath overlay architecture, while specializing each component to the requirements of the application and use case. Instead of generating a single overlay to support all possible changes to the application, our approach minimizes overhead using recurrent overlay generation to generate new overlays as application functionality shifts. Since any application can be decomposed into an FSM with datapath (referred to as an FSMD for simplicity), the presented overlay widens applicability to potentially any application and can potentially be integrated into any FPGA design tool that decomposes functionality into controllers and datapaths.

We demonstrate that our proposed overlay architecture can enable compilation times over 100,000 × faster than RTL designs. Although the overlay has an average area overhead of $8.4 \times$ at an average maximum clock speed of $285 \mathrm{MHz}$, computationally intensive benchmarks showed a lower area overhead of $3.5 \times$, which reduced further to $1.4 \times$ for the common use case of supporting multiple non-concurrent functions. In iterative development, we show recurrent overlay generation with our overlays provides faster development cycles than traditional FPGA application development after only two design iterations by amortizing overlay generation with rapid virtual compilations and reconfigurations, whose average reconfiguration time is 0.59 $\mu \mathrm{s}$. Even for use cases where the area and/or clock overhead is prohibitive, the approach can still be used to develop and debug the application, after which the developer can target the FPGA directly to maximize efficiency.

\section{RELATED WORK}

Existing research into overlay architectures has explored different degrees of coarse granularity in their processing elements and/or interconnect for a specific subset of applications. For example, intermediate fabrics [8] presented an overlay with a general island-styled layout of applicationlevel resources (e.g. adders, multipliers) for datapath-centric applications, whereas [5] introduced an island-styled overlay of 120 MIPS processors. Similarly, Deco [4] introduced a DSP block-based overlay with a linear-style interconnect to implement feed-forward pipelined datapaths whereas [3] introduced an overlay of application-level resources with an interconnect tailored to an application domain. Fewer studies have focused on control-intensive applications, such as FSM overlays [1] that virtualized FSM applications. Our proposed overlay differs from these approaches by introducing a new architecture that integrates control and datapath overlays to support potentially any application, while also enabling an iterative design process that automatically generates and tailors the architecture to requirements of an application under development.

Other work has also investigated automatic overlay generation [3]. That study presented a back-end synthesis approach for overlays that automatically generated virtual datapath architectures based on input source kernels. Although intended to similarly enable mainstream software design methodologies through fast and flexible compilation, the prior approach was limited by a less flexible overlay architecture that supported primarily datapath-centric applications with minimal control. By contrast, our proposed 


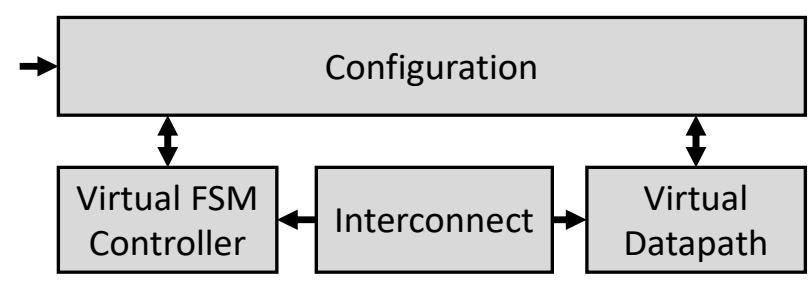

(a)

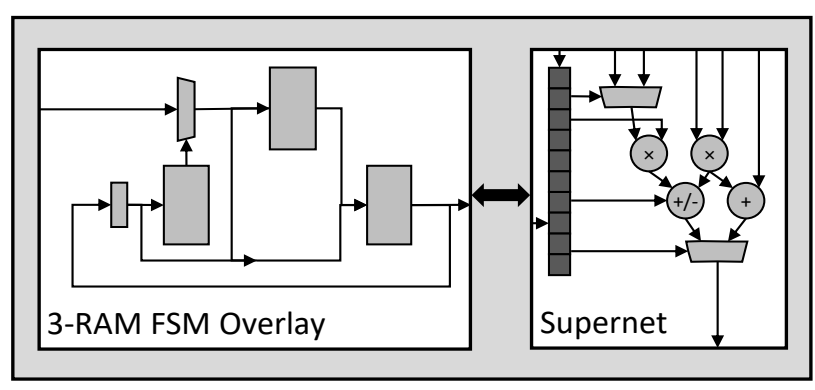

(b)

Figure 3. (a) An illustration of FSMD overlay architecture and (b) an example of generation creating an application-specialized version that integrates the 3-RAM FSM and supernet overlays.

approach introduces a more flexible overlay architecture to support arbitrary application development, which complements the previous work.

\section{RECURRENT OVERLAY GENERATION}

In this section, we present our envisioned usage of recurrently generated overlays to enable rapid development of FPGA applications. Note that a complete realization of this vision is well beyond the scope of a single paper, and will require efforts in architecture, HLS, design tools, and runtime resource management, which we collectively refer to as a framework for overlay-based application development. The approach presented in this paper (with technical details in Section 4) is a critical enabling technology for this framework that provides an appropriate overlay architecture to support numerous applications. We expect that future overlay research will build on top of the presented technology to move closer to the envisioned usage.

One challenge with overlay generation is that specializing an overlay for an application is critical for avoiding prohibitive overhead [2], [3]. However, specialization sacrifices flexibility, which is necessary for supporting changes that occur during application development. Although some flexibility can be built into an overlay, providing sufficient flexibility without knowledge of what changes may occur is difficult. To avoid this challenge, our approach focuses on recurrently regenerating overlays during application development and using reconfiguration to swap the new overlays into the FPGA as necessary.

In Figure 2(a), we illustrate an example overlay-based development process using recurrent overlay regeneration for multiple development iterations (labeled 1 through 6). Initially, the framework would generate an initial overlay (Overlay A) that can at least support the current operations of the code in Iteration 1, in addition to any other resources requested by the developer. Shown on the first timeline, the first compilation experiences no speedup due to the need to wait for Overlay A to be generated. After Overlay A is generated, an extended period of rapid compilation follows where Iterations 1 to 5 are rapidly compiled to Overlay A through fast virtual reconfiguration. By Iteration 6 , the application requirements change enough so that Overlay A can no longer support all requirements, at which point the framework must generate a new overlay (Overlay B). At this point, compilation again is slow due to overlay regeneration. Finally, after Overlay B is generated, fast compilation resumes for Iteration 6 and any future iterations, as long as the changes are still supported by Overlay B.

By contrast, Figure 2(b) presents a second timeline for existing FPGA application development, which experiences slow compilation for every iteration. Although FPGA compilation may be shorter than overlay regeneration due to less logic, the application developer will have likely completed far fewer iterations in the same period of time as our overlay approach. For example, by the time Iteration 2 is compiled in the FPGA timeline, the overlay has implemented Iterations 1 to 5 and has begun to compile Overlay B. As long as the overlays provide sufficient flexibility to amortize lengthy overlay generations over multiple iterations of rapid compilation, then overlay-based application development will provide significant productivity improvements. In fact, as long as the majority of development iterations are supported by existing overlays, this approach enables runtime hardware compilation [2], [3]. Enabling efficient flexibility is therefore a key challenge in overlay generation.

Although this topic of efficient flexibility remains an open question outside the scope of the paper, we evaluate experiments in Section 5 that provide initial feedback on tradeoffs between flexibility and area to support multiple FSMDs of an application in the same overlay, which can enable developers to rapidly explore tradeoffs in the application's design space that are supported by the overlay.

\section{FSMD OVERLAY ARCHITECTURE}

In this section, we present a new unified overlay architecture that integrates control and datapath overlays to widen applicability to any application that can be decomposed into an FSMD. As shown in Figure 3(a), the architecture is divided into four main parts: a virtual FSM architecture, a virtual datapath architecture, an interconnect between the two, and a configuration architecture. We then describe how the architecture can be generated and specialized from an application's requirements and/or developer specifications. As an example, Figure 3(b) illustrates a potential overlay architecture generated during the recurrent overlay generation process using a 3-RAM FSM overlay [1] and a supernet datapath overlay [3].

\subsection{Virtual FSM Controller}

To virtualize control logic, our unified overlay architecture adopts existing FSM overlays to implement FSMs within various parameter ranges. These overlays are memory-based architectures that map FSM logic in memories to enable runtime compilation. Acting as a coarsegrained lookup table, these memories can implement functions by using the function's inputs as an address and producing the function's output with the corresponding data. Since memory requirements grow exponentially with number of inputs, these reconfigurable architectures pay special 


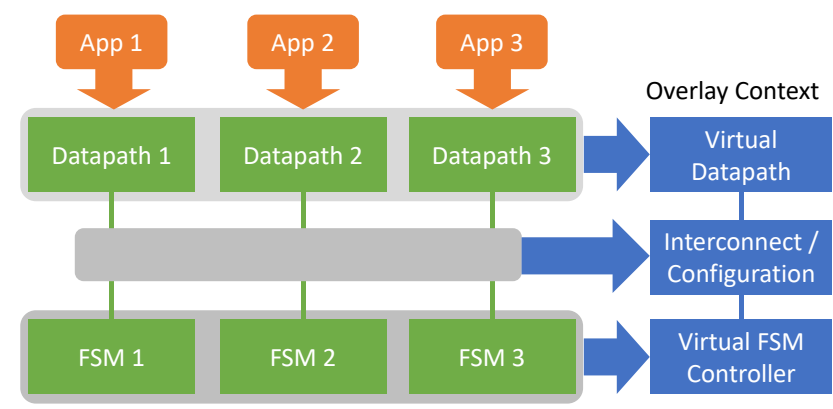

Figure 4. An illustration of overlay generation.

attention to how the FSM function is mapped onto memories. In many scenarios, these architectures partition the FSM function into sub-functions that are mapped onto a network of memories rather than a single memory [1]. Although this decomposition imposes different architectural restrictions, the resulting architecture has a lower memory imprint. The virtual FSM controller may therefore be programmed during runtime by changing the contents of the memory during execution through memory write ports. Due to the Paretooptimal tradeoffs between control requirements and area/clock costs, we evaluate our unified architecture using both a 3-RAM FSM overlay [1] and a Multi-RAM FSM overlay [9], which an overlay framework may select between based on an application's control requirements.

\subsection{Virtual Datapath}

To virtualize datapath logic, our unified architecture uses a reconfigurable datapath architecture derived from existing work. Past research in reconfigurable datapaths has generally focused on specialization and flexibility tradeoffs in the coarse-grained resources and interconnect. For resources, several works have focused on general-purpose softprocessor based overlays [5], whereas others have focused on application-level resources, such as adders and multipliers [4]. For interconnects, earlier work focused on island-style layouts [8], whereas more recent work has focused on tailored interconnects based on an application domain [3].

In our unified architecture, we use a virtual datapath architecture similar to the one described in [3] that relies on a tailored interconnect generated through datapath merging to minimize the overall overhead at the expense of flexibility. In such an approach, datapath netlists are extracted from input applications and are iteratively merged to form a supernet (a superset of netlists). Using this merged netlist, the supernet overlay produces a reconfigurable datapath with a tailored interconnect that considers the functional and communication similarities between applications. Since these architectures are generally created from known applications or preencoded domain knowledge, tailored overlay layouts achieve $2.6 \times$ to $8.9 \times$ lower area than general-purpose layouts [3]

\subsection{Interconnect}

One key difference from previous work is that our unified architecture integrates separate control and datapath overlays to provide more functionality. We employ a static interconnect between the virtual FSM and datapath consisting of control output signals that configure datapath elements on a state-by-state basis and control input signals from the datapath that affect a state transition. Based on common strategies in existing overlays, it may seem beneficial to use a reconfigurable network between the controller and datapath to enable runtime-configurable control connections. However, such a network would incur additional overhead. To avoid this overhead, we observed that the FSM overlay in the proposed architecture can already provide this reconfigurable connectivity with functional decomposition.

In both considered FSM overlays, the FSM sub-function decomposition yields separate input and output logic functions, which decouple the state-transition logic from the controller I/O. The input-logic function determines which inputs from the datapath are routed to the state-transition function, while the output-logic function determines the values of outputs to the datapath that correspond to a given state transition. As such, the overlay architecture may modify the connectivity between the virtual FSM and datapath by changing the contents of the memories that implement the input and output logic functions. As a result, our unified overlay architecture provides reconfigurable control connections without any additional overhead.

\subsection{Configuration}

For configuration, we leverage, extend, and combine the configuration architectures of the FSM overlays and datapath overlays from previous work. The FSM overlays use a simple controller circuit that loads configuration bits from a block RAM into the different RAMs inside the overlay. For the datapath overlay, previous work used a shift-register chain similar to an FPGA to load configuration information (e.g., mux select values, constants, etc.). Although we preserve this shift-register configuration in the datapath overlay, many inputs to the datapath now come from the FSM since the unified architecture is intended to support arbitrary FSMDs as opposed to circuits with limited control. As such, the configuration logic is split between the FSM and datapath overlays based on application needs. For datapath logic that can change on a state-by-state basis, the configuration is driven by the virtual FSM and must be programmed into the FSM overlay through the write ports in the FSM overlay memories. For datapath logic that remains static during execution (e.g. constants), the configuration is driven by configuration shift registers in the virtual datapath.

\subsection{Overlay Generation}

Figure 4 illustrates the different steps of overlay generation. The first step is decomposing the input applications into FSMDs through HLS. To provide extra flexibility for changes and design-space exploration, we use different HLS algorithms to construct different types of FSMDs and then determine the architectural requirements of the generated overlay from those FSMDs. Although we perform this decomposition manually in this paper for the purpose of evaluating the overlay architecture, we intend to integrate with different HLS tools and explore the impact of different HLS algorithms as future work. For example, the framework may add extra resources and generalize existing resources to support wider circuit flexibility.

A virtual datapath overlay is then automatically generated from application datapaths through datapath merging, as described in Section 4.2. In this process of accretion, source datapaths are iteratively merged together such that as few operators and nets are produced in the resulting overlay. The 
Table 1. Comparison of FSMD overlay and RTL compilation times, along with the necessary number of rapid compilations to amortize overlay generation times.

\begin{tabular}{|c|c|c|c|c|c|c|c|}
\hline \multirow[b]{2}{*}{ Kernel } & \multicolumn{3}{|c|}{$\frac{\text { Compilation Time }}{\text { (ms) }}$} & \multicolumn{2}{|c|}{$\begin{array}{c}\frac{\text { Speedup }}{\text { (Compared to RTL) }} \\
\text { (C) }\end{array}$} & \multicolumn{2}{|c|}{$\frac{\text { Amortization }}{\text { Factor }}$} \\
\hline & 3-RAM & M-RAM & RTL & 3-RAM & M-RAM & 3-RAM & M-RAM \\
\hline barcode1 & 1.9 & 1.3 & $177 \mathrm{~K}$ & $95 \mathrm{~K}$ & $137 \mathrm{~K}$ & 1.1 & 1.5 \\
\hline barcode 2 & 1.4 & 1.3 & $149 \mathrm{~K}$ & $103 \mathrm{~K}$ & $111 \mathrm{~K}$ & 1.6 & 1.4 \\
\hline barcode3 & 1.5 & 1.5 & $181 \mathrm{~K}$ & $117 \mathrm{~K}$ & $125 \mathrm{~K}$ & 1.1 & 1.1 \\
\hline diffeq1 & 1.1 & 0.9 & $225 \mathrm{~K}$ & $210 \mathrm{~K}$ & $251 K$ & 0.9 & 0.9 \\
\hline diffeq2 & 1.1 & 0.9 & $221 K$ & $208 \mathrm{~K}$ & $245 \mathrm{~K}$ & 1.0 & 1.0 \\
\hline diffeq3 & 1.1 & 0.9 & $210 \mathrm{~K}$ & $197 \mathrm{~K}$ & $231 K$ & 1.1 & 1.1 \\
\hline ellip1 & 1.1 & 1.0 & $202 \mathrm{~K}$ & $184 \mathrm{~K}$ & $208 \mathrm{~K}$ & 1.0 & 1.0 \\
\hline ellip2 & 1.1 & 1.0 & $202 \mathrm{~K}$ & $180 \mathrm{~K}$ & $211 \mathrm{~K}$ & 1.0 & 1.0 \\
\hline ellip3 & 1.1 & 1.0 & $200 \mathrm{~K}$ & $184 \mathrm{~K}$ & $206 \mathrm{~K}$ & 1.1 & 1.1 \\
\hline $\operatorname{gcd} 1$ & 1.8 & 1.1 & $192 \mathrm{~K}$ & $109 \mathrm{~K}$ & $169 \mathrm{~K}$ & 1.1 & 1.1 \\
\hline $\operatorname{gcd} 2$ & 1.0 & 1.0 & $192 \mathrm{~K}$ & $199 \mathrm{~K}$ & $194 K$ & 1.0 & 1.0 \\
\hline $\operatorname{gcd} 3$ & 1.4 & 1.3 & $189 \mathrm{~K}$ & $134 \mathrm{~K}$ & $149 \mathrm{~K}$ & 1.1 & 1.1 \\
\hline tlc1 & 0.6 & 0.7 & $144 \mathrm{~K}$ & $239 \mathrm{~K}$ & $221 \mathrm{~K}$ & 1.3 & 1.3 \\
\hline $\mathrm{tcl} 2$ & 0.7 & 0.8 & $144 \mathrm{~K}$ & $203 \mathrm{~K}$ & $182 \mathrm{~K}$ & 1.3 & 1.3 \\
\hline $\mathrm{tcl} 3$ & 1.2 & 1.2 & $145 \mathrm{~K}$ & $125 \mathrm{~K}$ & $123 \mathrm{~K}$ & 1.4 & 1.4 \\
\hline Average & 1.2 & 1.0 & $185 \mathrm{~K}$ & $166 \mathrm{~K}$ & $184 \mathrm{~K}$ & 1.1 & 1.2 \\
\hline
\end{tabular}

framework then analyzes the virtual datapath's configuration requirements and distributes the configuration logic to the datapath or FSM overlay based on application needs, as described in Section 4.4. The framework then generates a static interconnect between the datapath and FSM overlays that consists of these FSM overlay-driven outputs and of datapath outputs that can cause state transitions. Afterwards, different FSM parameters are extracted from the FSMs to automatically generate an appropriate FSM overlay. The first set of parameters are architecture independent: the numbers of inputs, outputs, and states that are supported in the FSM overlay. The second set of parameters are architecture dependent based on the chosen FSM overlay [1], [9].

\section{EXPERIMENTS}

\subsection{Experimental Setup}

With Pareto-optimal tradeoffs between existing FSM overlays, we evaluated our proposed architecture using FSM overlays from [1] and [9] with the datapath overlay from Section 4.2, labeling these as 3-RAM and M-RAM FSMD overlay, respectively. For benchmarks, since we envision overlays as a target architecture for HLS, we selected a subset of benchmarks from 1992 and 1995 HLS workshops [7], for which we manually created different FSMD circuits (labeled 1-3) with different Pareto-optimal tradeoffs. For future work, we intend to completely automate the process through integration with HLS tools, but such integration requires massive effort that is not necessary to evaluate the benefits of the presented architecture. We compiled the overlays and RTL implementations of the circuits in Quartus Prime Pro 17.1 for an Arria 10 GX1150 and perform all experiments on a $3.47 \mathrm{GHz}$ Xeon X5690 with $96 \mathrm{~GB}$ of RAM.

\subsection{Compilation Time Speedup}

In this section, we analyze the productivity advantages of overlays compared to RTL using compilation-time speedup. In Table 1, we analyze the overlay speedup across the three different FSMDs for each example. Compilation Time presents the compilation times (in milliseconds) of the FSMD code onto overlays and of RTL code directly onto the FPGA. For overlays, compilation time refers to the time required to generate an application's overlay configuration from our intermediate FSMD representation. For RTL, compilation time refers to the time required by Quartus to run synthesis and fitting for each FSMD from VHDL code. Speedup compares the compilation speedup of overlays relative to RTL for both overlays. Amortization Factor compares the number of design iterations (i.e., compilations) that must be supported by an overlay to amortize the overlay generation times compared to compiling RTL for each iteration.

Compared to RTL, overlays had compilation times that were generally five orders of magnitude faster, with an average compilation time of $1.2 \mathrm{~ms}$ and $1.0 \mathrm{~ms}$ for the 3 RAM and M-RAM FSMD overlays, respectively. The average compilation time for the RTL code was 184s. In general, the M-RAM FSMD overlay had faster compilation times than the 3-RAM FSMD overlay, with exception to $\operatorname{gcdl}, \operatorname{gcd} 3$, and each tlc FSMD. This overall trend can be attributed to the M-RAM's generally smaller configuration size, whose configuration data can be generated faster. For small benchmarks, such as $g c d$ and $t l c$, the initial cost of the M-RAM's more complex FSM decomposition contributed to a larger configuration size than the 3-RAM.

Due to these significant speedups, the 3-RAM and MRAM FSMD overlays only need to support 1.1 and 1.2 average compilations, respectively, to provide productivity advantages over directly compiling RTL for each compilation. This low amortization factor is significant because it suggests that even without significant flexibility, recurrently generated overlays can provide improved productivity. Since adding extra flexibility enables more rapid compiles at the cost of longer generation times, we intend to investigate the impact of flexibility on amortization factor as future work. For diffeq1, both overlays had faster generation times than RTL, due to variation in compilation time through vendor tools. In general, overlay generation took a similar amount of time as compiling an RTL circuit, with an average generation time of $207 \mathrm{~s}$ and $210 \mathrm{~s}$ for $3-\mathrm{RAM}$ and M-RAM FSMD overlays, respectively, compared to RTL's average compilation time of $185 \mathrm{~s}$.

\subsection{Resource Overhead}

In this section, we analyze the virtualization costs of implementing an application on an overlay compared to a direct implementation in RTL. We present area in terms of ALMs as the dominant resource type and clock as the max frequency reported by Quartus. In Figure 5, we compare our two overlay architectures to RTL designs in two situations: (1) supporting a single FSMD of each application and (2) supporting all three FSMDs of each application. For the first scenario, we obtain the synthesis results for each FSMD of an application and present the average (labeled Single). Although no developer would implement multiple application FSMDs in the same design, for this analysis we then treat these FSMDs as different applications of the same domain that the overlays must all support (labeled Multi). Whereas an overlay would be virtually reconfigured to implement a different function, we model support for different functions in RTL as a selectively enabled circuit that 

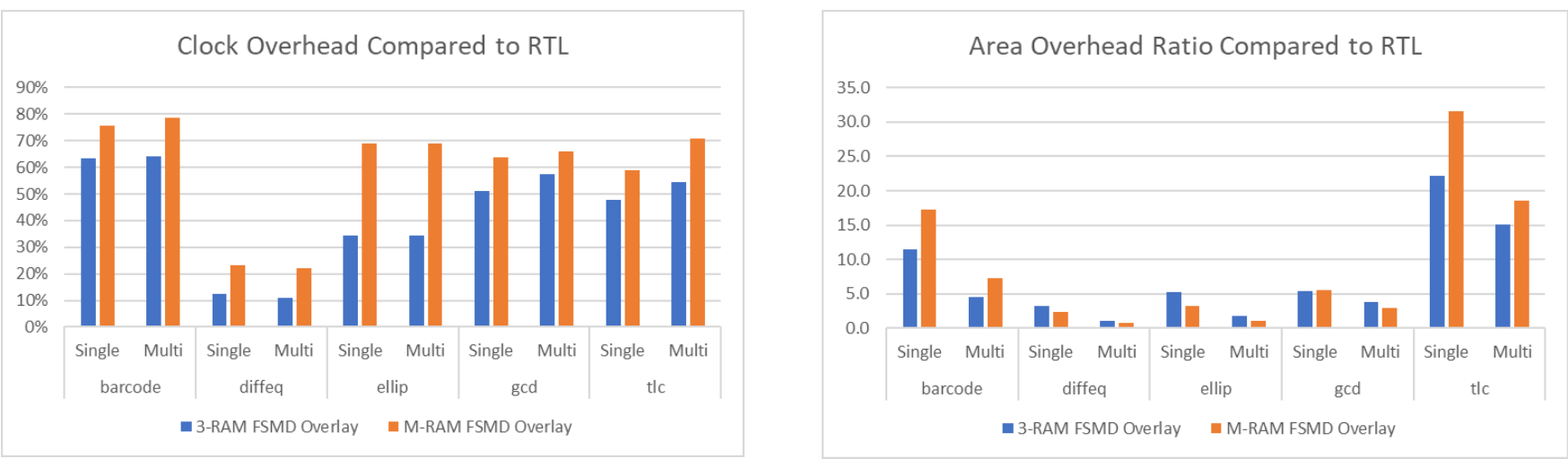

Figure 5. Comparison of overlay clock and area overhead for single- and multi-FSMD support.

implements all required functions. Although RTL code could leverage partial reconfiguration in a similar manner to reduce area requirements, partial reconfiguration is orders of magnitude slower than overlay reconfiguration [3].

Compared to RTL, both overlays had significant clock and area overhead, showing an average $43 \%$ clock degradation and an $8.4 \times$ area overhead for the 3-RAM FSMD Overlay, and an average $59 \%$ clock degradation and a $10.5 \times$ area overhead for the M-RAM FSMD Overlay. Despite these significant values, the results are however skewed by small FSMD circuit sizes. In practice, these circuits would be integrated with other components such as specialized IP and may not be on the critical path (with an average clock frequency close to $200 \mathrm{MHz}$ ) or contribute significantly to overall area (with less than $1 \%$ of total logic utilization).

For supporting a single FSMD in a design, we observe that overlays had lower area overhead on datapath-intensive circuits (diffeq, ellip) than on control-intensive circuits (barcode, gcd, tlc). In datapath-centric circuits, datapath elements constituted a significant portion of area for both overlays and RTL, which reduces the overhead of the overlay's reconfigurable logic. Although larger in controlintensive circuits, that overhead may not be practically prohibitive due to the small size of many control circuits. When supporting multiple FSMDs, overlays had similar clock degradations, but lower area overheads than their single FSMD counterparts due to the overlay's innate resource sharing through virtual reconfiguration that may not be present in a selectively enabled RTL circuit.

Compared to the M-RAM FSMD overlay, the 3-RAM FSMD overlay had a lower clock overhead in all tests due to decomposing the FSM across fewer memories than the MRAM FSMD architecture. For area, the 3-RAM FSMD Overlay had smaller area overhead in the control-intensive circuits and larger area overhead in the datapath-intensive circuits due to worse scalability with area requirements. Overall, the 3-RAM FSMD overlay had the higher average clock frequency of $597 \mathrm{MHz}$ compared to the M-RAM FSMD overlay's $536 \mathrm{MHz}$, and a larger average area of 285 ALMs compared to M-RAM FSMD overlay's 191 ALMs.

\section{CONCLUSION}

In this paper, we present a new FSMD-based overlay architecture that integrates datapath-centric overlays with FSM overlays to support arbitrary datapaths and controllers for more general FPGA application development. Compared to RTL applications, our proposed FSMD overlay enables $100,000 \times$ faster compilation at an average area overhead of $8.4 \times$ with an average maximum clock speed of $285 \mathrm{MHz}$ that is reduced to an area overhead of $1.4 \times$ in common overlay use cases. When integrated in a recurrent overlay generation process that automatically generates and specializes an overlay for an application, we show that development on overlays provides faster development cycles than on FPGAs after only two design iterations by amortizing overlay generation through fast virtual reconfigurations.

\section{Acknowledgements}

This work was supported by the National Science Foundation under grant CNS-1149285.

\section{REFERENCES}

[1] P. Cooke, L. Hao, and G. Stitt. Finite-state-machine overlay architectures for fast fpga compilation and application portability. ACM Trans. Embed. Comput. Syst., 14(3):54:154:25, Apr. 2015.

[2] J. Coole and G. Stitt. Fast, flexible high-level synthesis from opencl using reconfiguration contexts. IEEE Micro, 34(1):4253, Jan 2014.

[3] J. Coole and G. Stitt. Adjustable-cost overlays for runtime compilation. In 2015 IEEE 23rd Ann. Int. Symp. on FieldProgrammable Custom Computing Machines, pages 21-24, May 2015

[4] A. K. Jain, X. Li, P. Singhai, D. L. Maskell, and S. A. Fahmy. Deco: A dsp block based fpga accelerator overlay with low overhead interconnect. In 2016 IEEE 24th Ann. Int. Symp. on Field-Programmable Custom Computing Machines, pages 18, May 2016.

[5] C. Kumar HB, P. Ravi, G. Modi, and N. Kapre. 120-core microaptiv mips overlay for the terasic de5-net fpga board. In Proc. of the 2017 ACM/SIGDA Int. Symp. on FieldProgrammable Gate Arrays, pages 141-146. ACM, 2017

[6] B. E. Nelson, M. J. Wirthlin, B. L. Hutchings, P. M. Athanas, and S. Bohner. Design productivity for configurable computing. In ERSA 2008, Las Vegas, Nevada, USA, July 14 17, 2008, pages 57-66, 2008.

[7] P. R. Panda and N. D. Dutt. 1995 high level synthesis design repository. In Proc. of the 8th Int. Symp. on Syst. Synthesis, ISSS '95, pages 170-174, New York, NY, USA, 1995. ACM.

[8] G. Stitt and J. Coole. Intermediate fabrics: Virtual architectures for near-instant fpga compilation. IEEE Embed. Syst. Letters, 3(3):81-84, Sept 2011.

[9] D. Wilson and G. Stitt. A scalable, low-overhead finite-state machine overlay for rapid FPGA application development. CoRR, abs/1705.02732, 2017 